\title{
Addendum to: Multiplicity of critical points in presence of a linking: application to a superlinear boundary value problem, NoDEA. Nonlinear Differential Equations Appl. 11 (2004), no. 3, 379-391, and a comment on the generalized Ambrosetti- Rabinowitz condition
}

Dimitri Mugnai

\begin{abstract}
We show the incompleteness of a usually used version of the generalized Ambrosetti-Rabinowitz condition in superlinear problems, also used in the paper cited in the title, and we propose a complete one.

Mathematics subject classification (2000). 35J20, 35J61.

Keywords. Generalized Ambrosetti-Rabinowitz condition, Superlinear equations.
\end{abstract}

\section{Introduction}

Since the appearing of that milestone in partial differential equations given by the paper by Ambrosetti and Rabinowitz where the Mountain Pass was introduced (see [1]), thousands of papers have studied semilinear problems like

$$
\begin{cases}-\Delta u=g(x, u) & \text { in } \Omega, \\ B u=0 & \text { on } \partial \Omega,\end{cases}
$$

where $\Omega$ is a domain of $\mathbb{R}^{N}, N \geq 3$, and $B$ is a boundary operator, for example the Dirichlet or the Neumann one. $\Omega$ is allowed also to be unbounded, with obvious adaptations. 
Moreover, also related quasilinear versions, for example in presence of the $p$-Laplacian operator $\Delta_{p} u=\operatorname{div}\left(|D u|^{p-2} D u\right), p \in(1, \infty)$,

$$
\begin{cases}-\Delta_{p} u=g(x, u) & \text { in } \Omega, \\ B u=0 & \text { on } \partial \Omega,\end{cases}
$$

have been widely studied.

In order to study problem (1) when $\Omega$ is bounded, just to fix the ideas, the usual assumptions, introduced in [1], are:

(i) $g: \bar{\Omega} \times \mathbb{R} \rightarrow \mathbb{R}$ is (locally Hölder) continuous,

(ii) $g$ is subcritical in the sense of Sobolev's Embedding Theorem at infinity,

(iii) $g(x, s)=o(|s|)$ as $s \rightarrow 0$ uniformly in $\Omega$,

(iv) the now-called Ambrosetti-Rabinowitz condition holds: there exist $\mu>2$ and $R \geq 0$ such that

$$
0<\mu \int_{0}^{s} g(x, t) d t \leq g(x, s) s \quad \text { for any }|s|>R \text { and } x \in \bar{\Omega} .
$$

Since then, there have been a plenty of papers where the authors consider problem (2) with $g: \Omega \times \mathbb{R} \rightarrow \mathbb{R}$, possibly just a Carathéodory function, satisfying (ii) and (iii) and the following generalized Ambrosetti-Rabinowitz condition: there exist $\mu>p$ and $R \geq 0$ such that

$$
0<\mu \int_{0}^{s} g(x, t) d t \leq g(x, s) s \quad \text { for any }|s|>R \quad \text { and } \quad \text { for a.e. } x \in \Omega \text {. }
$$

At a first look the two conditions look pretty much the same, and in fact they are in the autonomous case $g(x, s)=g(s)$, but the consequences are extremely different, at least in view of the applications. Indeed, by direct integration, (3) implies that there exist $c_{1}>0$ and $c_{2} \geq 0$ such that

$$
G(x, s)=\int_{0}^{s} g(x, t) d t \geq c_{1}|s|^{\mu}-c_{2} \quad \text { for all } s \in \mathbb{R} \quad \text { and } \quad x \in \bar{\Omega} .
$$

In a massive number of papers it is written that integrating (3) - or (4) -, we get that there exist $c_{1}>0$ and $c_{2} \geq 0$ such that

$$
G(x, s) \geq c_{1}|s|^{\mu}-c_{2} \quad \text { for all } s \in \mathbb{R} \quad \text { and } \quad \text { a.e. } x \in \Omega .
$$

Not to be unfair, we only quote our [3], were such a mistake was done assuming (3) with $R=0$ and deducing (6) with $c_{2}=0$. Luckily such a mistake was not done in [2], a natural development of [3].

However, this deduction is false. Indeed, consider the function $g:(0, \pi) \times$ $\mathbb{R} \rightarrow \mathbb{R}$ defined as $g(x, s)=\sin x|s|^{\mu-2} s$; then it obviously verifies $\mu G(x, s) \leq$ $g(x, s) s$ in $\Omega$ for all $s$, but there are no $c_{1}>0, c_{2} \geq 0$ such that $G(x, s) \geq$ $c_{1}|s|^{\mu}-c_{2}$ in $\Omega$.

The mistake is simply in the integration and, we suppose, it is made just because the integral has not been really calculated. Indeed, (6) follows from (4) only if 


$$
\operatorname{ess} \inf _{x \in \Omega} G(x, \pm R)>0,
$$

a condition which is not satisfied by the example above, since (4) holds only in $\Omega$ and not in $\bar{\Omega}$.

However, it is well known that condition (6) is extremely important, for example, in order to verify mountain pass structures.

Moreover, also reversed forms of (4), like

$\mu \int_{0}^{s} g(x, t) d t \geq g(x, s) s>0 \quad$ for any $0<|s| \leq R \quad$ and $\quad$ for a.e. $x \in \Omega,(8)$

have been extensively used, for instance in order to compute critical groups of the associated action functional, deriving too fast that in this case there exists $c_{1}>0$ such that

$$
G(x, s) \geq c_{1}|s|^{\mu} \quad \text { for all }|s| \leq R \quad \text { and } \quad \text { a.e. } x \in \Omega,
$$

without knowing, again, that (7) holds.

In conclusion, working with functions satisfying (4), or (3) only in $\Omega$ and not in $\bar{\Omega}$, forces to add condition (6) to (3) or (4) and consider them as a unique hypothesis, as well as (9) should be assumed together with (8).

Therefore, in [3], where condition (3) was assumed with $R=0$, one must ignore Remark 1 , while condition (6) with $c_{2}=0$ must be taken as part of hypothesis $\left(g_{4}\right)$.

\section{References}

[1] Ambrosetti, A., Rabinowitz, P.H.: Dual variational methods in critical point theory and applications. J. Funct. Anal. 14, 349-381 (1973)

[2] Mugnai, D.: Asymptotic behaviour, nodal lines and symmetry properties for solutions of superlinear elliptic equations near an eigenvalue. ESAIM Control Optim. Calc. Var. 11(4), 508-521 (2005)

[3] Mugnai, D.: Multiplicity of critical points in presence of a linking: application to a superlinear boundary value problem. NoDEA Nonlinear Differ. Equ. Appl. 11(3), 379-391 (2004)

Dimitri Mugnai

Dipartimento di Matematica e Informatica

Università di Perugia

Via Vanvitelli 1

Perugia 06123

Italy

e-mail: mugnai@dmi.unipg.it

Received: 8 October 2010.

Accepted: 20 June 2011. 